\title{
Highly Uniform and Stable n-Type Carbon Nanotube Transistors by Using Positively Charged Silicon Nitride Thin Films
}

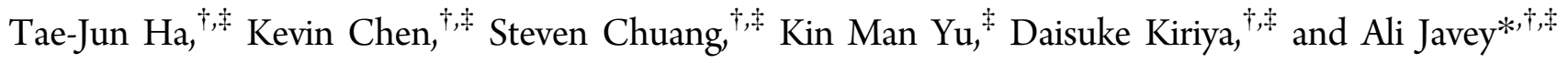 \\ ${ }^{\dagger}$ Electrical Engineering and Computer Sciences, University of California, Berkeley, California 94720, United States \\ ${ }^{\ddagger}$ Materials Sciences Division, Lawrence Berkeley National Laboratory, Berkeley, California 94720, United States
}

Supporting Information

ABSTRACT: Air-stable $\mathrm{n}$-doping of carbon nanotubes is presented by utilizing $\mathrm{SiN}_{x}$ thin films deposited by plasmaenhanced chemical vapor deposition. The fixed positive charges in $\mathrm{SiN}_{x}$, arising from ${ }^{+} \mathrm{Si} \equiv \mathrm{N}_{3}$ dangling bonds induce strong field-effect doping of underlying nanotubes. Specifically, an electron doping density of $\sim 10^{20} \mathrm{~cm}^{-3}$ is estimated from capacitance voltage measurements of the fixed charge within the $\mathrm{SiN}_{x}$. This high doping concentration results in thinning of
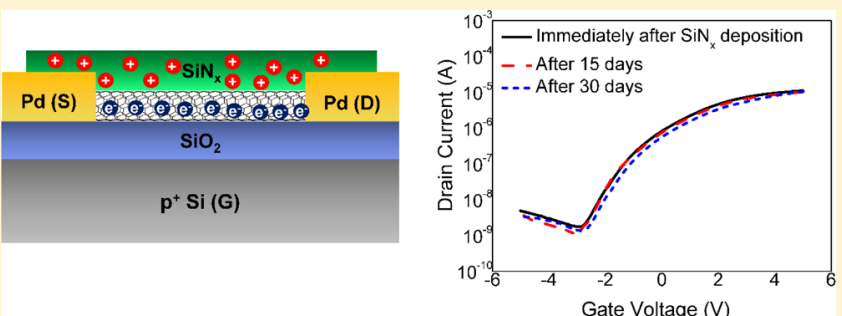
the Schottky barrier widths at the nanotube/metal contacts, thus allowing for efficient injection of electrons by tunnelling. As a proof-of-concept, n-type thin-film transistors using random networks of semiconductor-enriched nanotubes are presented with an electron mobility of $\sim 10 \mathrm{~cm}^{2} / \mathrm{V} \mathrm{s}$, which is comparable to the hole mobility of as-made p-type devices. The devices are highly stable without any noticeable change in the electrical properties upon exposure to ambient air for 30 days. Furthermore, the devices exhibit high uniformity over large areas, which is an important requirement for use in practical applications. The work presents a robust approach for physicochemical doping of carbon nanotubes by relying on field-effect rather than a charge transfer mechanism.

KEYWORDS: CMOS, surface doping, single-walled carbon nanotubes, field-effect transistors, $n$-FETs
$S_{\mathrm{e}}$ ingle-walled carbon nanotubes (SWCNTs) are promising electronic materials for thin film transistors (TFTs) due to their excellent electrical properties ${ }^{1-3}$ and ease of processing for large scale production. ${ }^{4-12}$ In particular, solution processed semiconductor-enriched nanotubes have been configured as high performance TFTs on mechanically flexible ${ }^{10,13}$ and rigid substrates ${ }^{5,14,15}$ with high ON/OFF current ratios ${ }^{16}$ and carrier mobilities. ${ }^{1}$ Given the high uniformity of the process, ${ }^{5}$ systemlevel applications have been explored, including active-matrix backplanes for driving flexible organic light-emitting diode displays $^{13,14,17}$ and large-area sensor networks capable of mapping external stimuli. ${ }^{18}$ Despite the tremendous progress in this field, to date most works have focused on p-type TFTs given the difficulty to form stable and robust electrical contacts to the conduction band of carbon nanotubes. However, for many practical applications, n-type transistors in addition to their p-type counterparts are needed to enable complementary metal-oxide-semiconductor (CMOS) circuits, thus lowering power consumption ${ }^{19}$ which is critical for large area systems. In this regard, a wide range of schemes have been explored in the past. A few examples include the use of low work function metal contacts ${ }^{20-23}$ for enhanced electron injection and surface charge transfer doping by electron withdrawing polymers ${ }^{24,25}$ or molecular species. ${ }^{26,27}$ However, many of these approaches suffer from insufficient air stability for reliable long-term operation ${ }^{19}$ although significant improvements have been made through encapsulation. ${ }^{28,29}$ As an alternative, metal oxide encapsulation ${ }^{30-32}$ has been recently reported to electron- dope nanotubes, presenting one potentially promising approach.

Here, we explore "physicochemical field-effect" n-doping of carbon nanotubes by silicon nitride $\left(\mathrm{SiN}_{x}\right)$ thin films. $\mathrm{SiN}_{x}$ deposited by plasma-enhanced chemical vapor deposition (PECVD) is well-known to exhibit positive fixed charges. ${ }^{33-35}$ These fixed charges arise from ${ }^{+} \mathrm{Si} \equiv \mathrm{N}_{3}$ dangling bonds within the nitride, known as $\mathrm{K}^{+}$centers, ${ }^{36}$ and the sheet density can be controllably tuned to between $10^{11}-10^{13} \mathrm{~cm}^{-2}$ by changing the deposition conditions or conducting post deposition treatments. ${ }^{34,37}$ This property of $\mathrm{SiN}_{x}$ has been used in the past to induce surface band bending of $\mathrm{Si}$ solar cells, ${ }^{38-40}$ thus providing a robust surface passivation scheme for reduced surface recombination of minority carriers. More recently, we adopted this concept to controllably n-dope two-dimensional (2D) semiconductors, such as $\mathrm{WSe}_{2} \cdot{ }^{35}$ Unlike surface charge transfer doping that is often explored for nanoscale inorganic and organic materials, the fixed charges in $\mathrm{SiN}_{x}$ induce fieldeffect doping of the underlying semiconductor. Because the fixed charge density can be as high as $10^{13} \mathrm{~cm}^{-2}$, high doping levels at the surface of the underlying semiconductor can be achieved. Here, by depositing $\mathrm{SiN}_{x}$ thin films on top of carbon nanotubes, we are able to obtain n-type transistors with comparable mobilities and $\mathrm{ON}$ currents as their p-type

Received: September 26, 2014

Revised: November 26, 2014

Published: December 1, 2014 

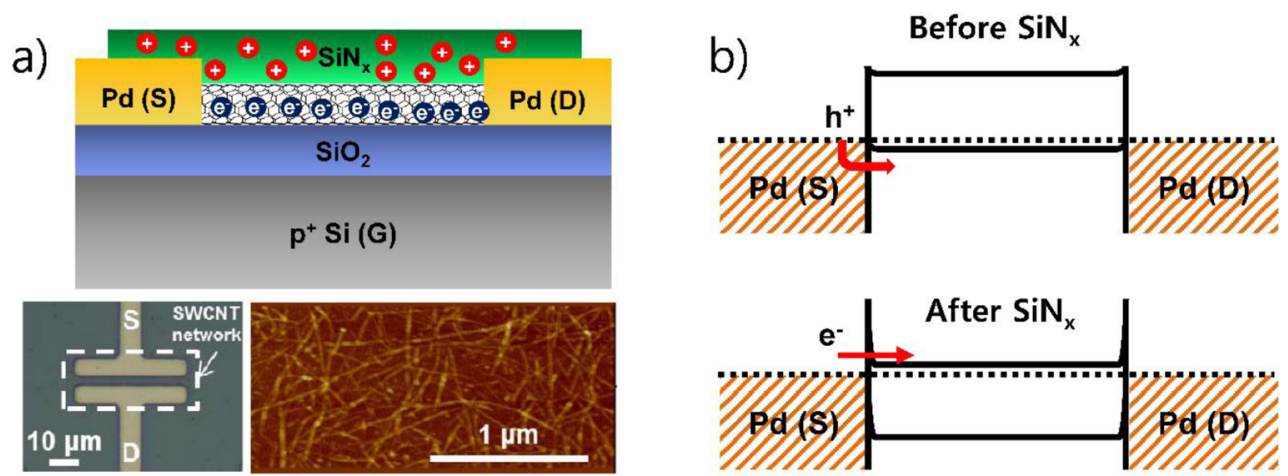

Figure 1. $\mathrm{SiN}_{x}$ field-effect doping of carbon nanotubes. (a) Cross-sectional schematic of a SWCNT TFT n-doped with a thin film of SiN ${ }_{x}$ (top). An optical microscope image of a representative transistor (bottom left) and an AFM image of the SWCNT network inside the channel (bottom right) are also shown. (b) Qualitative band diagram of a nanotube transistor in the ON-state before (top) and after (bottom) SiN $_{x}$ field-effect doping.
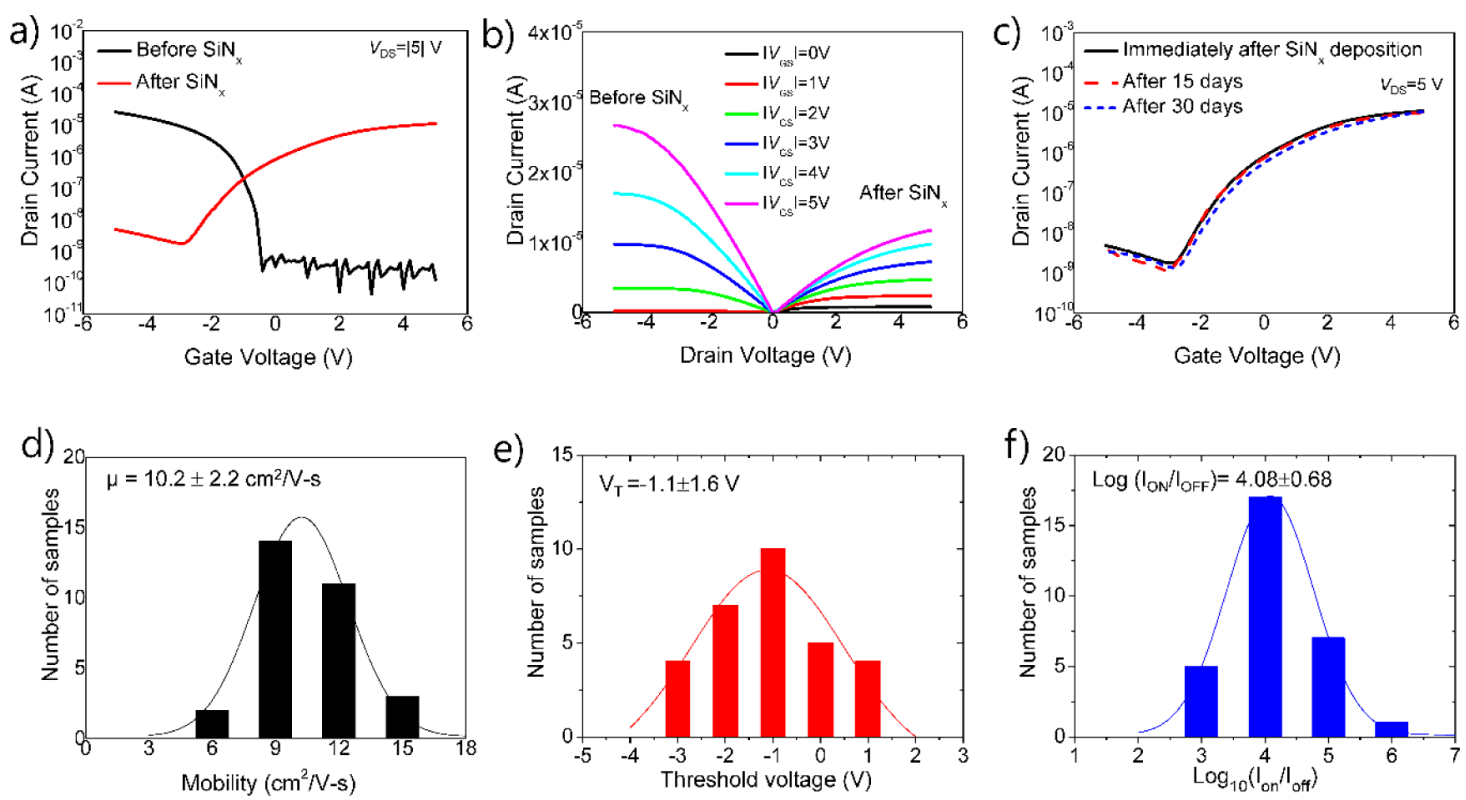

Figure 2. SWCNT n-type TFTs. (a) Transfer and (b) output characteristics of a representative SWCNT TFT (gate length of $4 \mu \mathrm{m}$ and width of 50 $\mu \mathrm{m}$ ) before and after $\mathrm{SiN}_{x}$ deposition. (c) Transfer characteristics of an n-type TFT immediately after $\mathrm{SiN}_{x}$ deposition (solid black curve), as well as after 15 (dashed red curve) and 30 days (dashed blue curve), showing virtually no change in performance. Histograms of the (d) electron mobility, (e) threshold voltage, and (f) log of the ON/OFF current ratio for 30 n-type TFTs.

counterparts, and ON/OFF current ratios of up to $10^{6}$. In particular we demonstrate n-type transport behavior for both single nanotube and networked transistors with high yield and long-term stability in air. We also demonstrate the compatibility of our approach for CMOS logic by fabricating a proof-ofprinciple inverter based on p-and n-type SWCNT-TFTs on the same substrate through patterned deposition of $\mathrm{SiN}_{x}$.

The effect of $\operatorname{SiN}_{x}$ on the electrical properties of nanotubes is first examined by fabricating TFTs based on random networks of semiconductor-enriched SWCNTs. SWCNT TFTs (Figure 1a) are fabricated on a degenerately p-doped silicon substrate that functions simultaneously as the back gate electrode with a $50 \mathrm{~nm}$ thick thermally grown silicon dioxide $\left(\mathrm{SiO}_{2}\right)$ serving as the gate dielectric. After cleaning in acetone, methanol, and isopropyl alcohol, the $\mathrm{SiO}_{2}$ surface was treated with an oxygen plasma at $120 \mathrm{~W}$ for $2 \mathrm{~min}$. Poly-L-lysine $\left(0.1 \% \mathrm{w} / \mathrm{v}\right.$ in $\mathrm{H}_{2} \mathrm{O}$, Sigma-Aldrich) solution was then drop-cast onto the $\mathrm{SiO}_{2}$ for 5 min and subsequently rinsed in deionized water to functionalize the surface for SWCNT deposition. Then, 99\% semiconducting SWCNT suspension (NanoIntegris Inc.) was deposited by drop-casting for $10 \mathrm{~min}$ followed by a rinse with DI water to remove the excess nanotube solution. This results in a densely packed SWCNT network with a density on the order of $\sim 30-$ 60 nanotubes per square micron on the poly-L-lysine treated $\mathrm{SiO}_{2}$ surface as shown in the atomic force microscope (AFM) image of Figure 1a. Assuming an average diameter of $1.4 \mathrm{~nm}$ and length of $1 \mu \mathrm{m}$, this corresponds to a surface coverage area of $\sim 4-9 \%$. Source/drain (S/D) contacts were patterned by photolithography, electron-beam evaporation of $\mathrm{Ti} / \mathrm{Pd}(0.5 / 40$ $\mathrm{nm})$ thin films, and lift-off of the resist in acetone. Subsequently, the SWCNT network was patterned to confine them to the channel region (i.e., space between the $S / D$ electrodes) by using photolithography and $\mathrm{O}_{2}$ plasma etch. To dope the samples, $\mathrm{SiN}_{x}$ was deposited via PECVD using an Oxford Plasmalab 80plus PECVD System operating at a frequency of $13.56 \mathrm{MHz}$. The deposition process was kept the same for all runs with a process temperature of $150{ }^{\circ} \mathrm{C}$, power density of $40 \mathrm{~mW} / \mathrm{cm}^{2}$, and a pressure of $900 \mathrm{mTorr}$ with $10 \%$ $\mathrm{SiH}_{4}$ (diluted in $\mathrm{Ar}$ ) at $100 \mathrm{sccm}$ and $\mathrm{NH}_{3}$ at $30 \mathrm{sccm}$. As the $\mathrm{K}^{+}$centers are expected to reside within the first $20 \mathrm{~nm}$ of the 

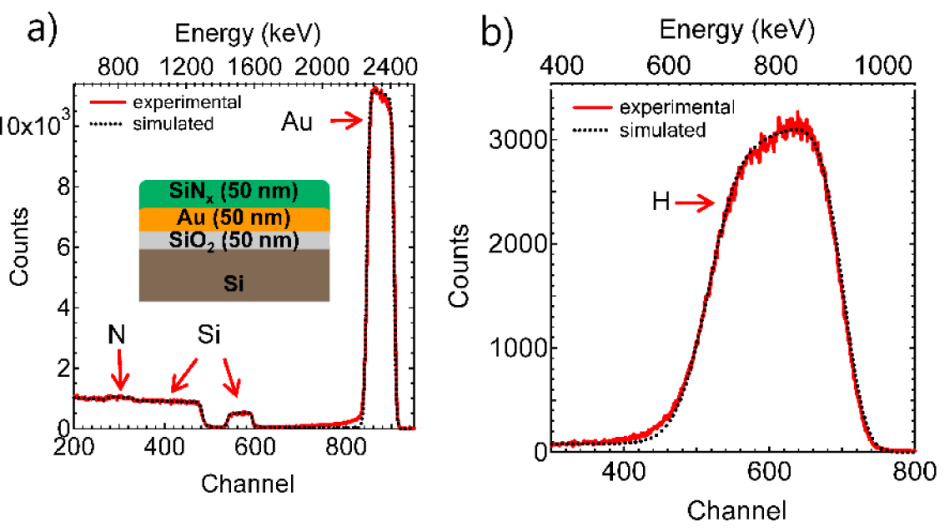

C)

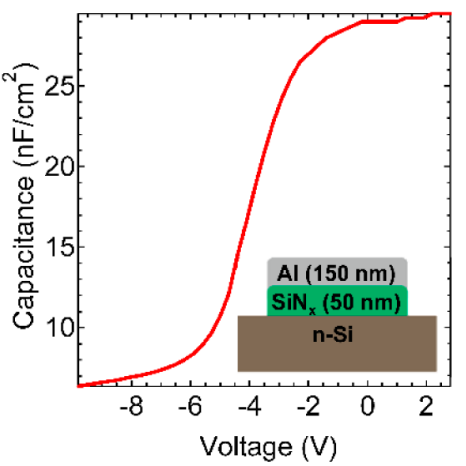

Figure 3. Material characterization of $\mathrm{SiN}_{x}$ thin films. (a) RBS spectrum of $\mathrm{SiN}_{x}$ deposited on $\mathrm{Au}$ on a $\mathrm{Si} / \mathrm{SiO}_{2}$ wafer. (b) Hydrogen distribution within the sample obtained from hydrogen forward scattering. The simulated fit to the experimental data from SINMRA codes yields a stoichiometry of $\mathrm{Si}_{0.39} \mathrm{~N}_{0.35} \mathrm{H}_{0.26}$. (c) Capacitance-voltage measurement of the MIS structure shown in the inset.

$\mathrm{SiN}_{x}$ film interface, ${ }^{40}$ a deposition time of $340 \mathrm{~s}$ was used to obtain a film thickness of $\sim 100 \mathrm{~nm}$ for all samples to ensure high uniformity from device-to-device and batch-to-batch. No change in the electrical properties was observed for $\mathrm{SiN}_{x}$ thicknesses of $>100 \mathrm{~nm}$ (Supporting Information Figure S1).

Figure $1 \mathrm{~b}$ shows the qualitative band diagrams for a SWCNT device before and after $\mathrm{SiN}_{x}$ deposition, depicting the contact properties for charge injection. It should be noted that polarity (n-type versus p-type) of transistors is governed by the properties of the electrical contacts rather than the channel doping type. Specifically, the source contact determines which carrier type can be injected into the channel. In the ON-state of a p-type transistor, the source contact provides injection of holes into the channel while electrons are injected for the case of an n-type device. Before $\mathrm{SiN}_{x}$ deposition, the Schottky barrier (SB) height to the valence band of SWCNTs is small due to the use of high work function Pd S/D contacts, thus allowing for efficient hole injection while presenting a large SB height to the conduction band for electron injection. Thereby, as-made devices exhibit p-type characteristics. After $\operatorname{SiN}_{x}$ deposition, the positive fixed charges within $\mathrm{SiN}_{x}$ are expected to induce field-effect doping of the underlying SWCNTs. By using the optimal stoichiometry, the fixed positive charge density in $\mathrm{SiN}_{x}$, corresponding to the electron doping level in SWCNTs, can be high enough to sufficiently thin the SBs to the conduction band, thereby allowing for efficient electron tunneling from the contacts into nanotubes. Thus, n-type transport can be expected for $\mathrm{SiN}_{x}$ coated devices.

Figure 2a,b shows the transfer (drain current, $I_{\mathrm{DS}}$, versus gate voltage, $V_{\mathrm{GS}}$ ) and output (drain current, $I_{\mathrm{DS}}$, versus drain voltage, $V_{\mathrm{DS}}$ ) characteristics of a representative SWCNT TFT with a channel length and width of 4 and $50 \mu \mathrm{m}$, respectively. The device is electrically measured before and after $\operatorname{SiN}_{x}$ deposition. Clear polarity conversion from $\mathrm{p}$ - to $\mathrm{n}$-type transport is observed upon $\mathrm{SiN}_{x}$ deposition. The electrical properties are nearly symmetric for both cases with the ONcurrent for the $\mathrm{n}$-channel transport being $\sim 2 \times$ lower than that of the p-channel prior to $\mathrm{SiN}_{x}$. The linear behavior of the output characteristics at low $V_{\mathrm{DS}}$ (Figure $2 \mathrm{~b}$ ) for the n-TFT suggests efficient injection of electrons at the source contact, which is facilitated by sufficient thinning of the SBs by $\mathrm{SiN}_{x}$ doping as depicted in the band diagram of Figure 1b. At the same time, the doping level is still at a level to allow for gate modulation of the conductance of ultrathin nanotubes with
$I_{\mathrm{ON}} / I_{\mathrm{OFF}} \sim 10^{4}$ (Figure 2a). As a result, high performance $\mathrm{n}$ type TFTs are obtained.

Importantly, the obtained n-type TFTs exhibit excellent airstability. Figure $2 c$ shows the transfer characteristics of a device immediately after $\mathrm{SiN}_{x}$ deposition as well as after 15 and 30 days in ambient air. Owing to the robustness of the silicon nitride, the device is highly stable in air without any noticeable change in the electrical properties. This is in distinct contrast to the surface charge transfer doped nanotubes obtained by deposition of molecular ${ }^{26}$ species on the surface. In addition to air stability, the devices also exhibit similar levels of stability when stressed under device operation conditions (i.e., bias stressing) compared to their undoped counterparts (Supporting Information Figure S2). Such stability is essential for any practical device technology and presents an important advance in the field of carbon nanotube electronics. The use of inorganic nitride rather than organic species presents a more robust platform for the fabrication of complex systems over large-areas, where multiple process steps are required.

The uniformity of $\mathrm{SiN}_{x}$ doping, and the enabled devices, is essential for exploring practical applications. The electrical properties of 30 SWCNT TFTs coated with $\mathrm{SiN}_{x}$ are measured and analyzed. The average and standard deviation of mobility, threshold voltage, and log ON/OFF current ratio are extracted as $10.2 \pm 2.2 \mathrm{~cm}^{2} / \mathrm{V}$-s (Figure $2 \mathrm{~d}$ ), $-1.1 \pm 1.6 \mathrm{~V}$ (Figure $2 \mathrm{e}$ ), and $4.08 \pm 0.68$ (Figure $2 \mathrm{f}$ ), respectively. Notably, the $\sim 20 \%$ variation in mobility of our n-type TFTs is similar to that of the as-made p-type TFTs, ${ }^{13}$ suggesting that $\mathrm{SiN}_{x}$ field-effect doping does not induce further device-to-device variation. The obtained device uniformity is respectable given that the fabrication processing was performed in an academic laboratory, and yet sufficient for exploring certain system-level applications, such as active-matrix back-planes for driving sensor arrays $^{18}$ or displays. ${ }^{13}$

The effect of metal contacts on the electrical properties of $\mathrm{SiN}_{x}$ coated SWCNT TFTs is also characterized. TFTs with $\mathrm{Pd}, \mathrm{Au}, \mathrm{Al}$, and $\mathrm{Ni}$ as the $\mathrm{S} / \mathrm{D}$ contacts are fabricated. In all cases, $\mathrm{n}$-type transistor behavior is obtained regardless of the metal contact choice (Supporting Information Figure S3). However, Pd contacted devices exhibit the highest ON-state current densities, corresponding to the lowest contact resistance for electron injection after $\mathrm{SiN}_{x}$ deposition. Although $\mathrm{Pd}$ has the highest work function among the metals explored here and thus results in the largest SB height for electrons, it still provides the lowest contact resistance for electron injection 
a)

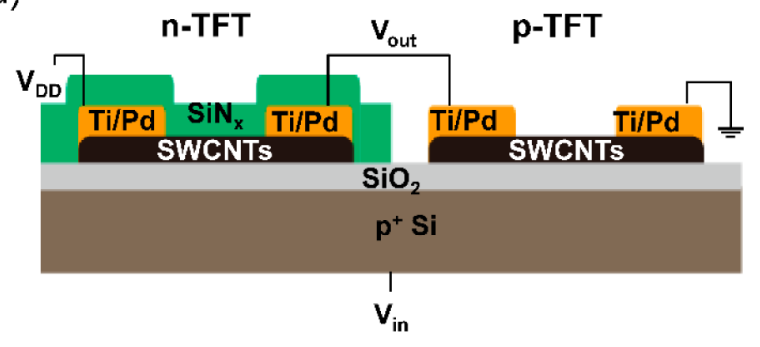

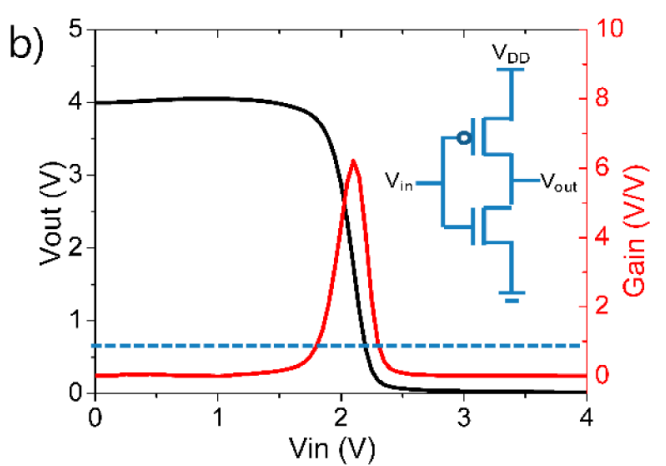

Figure 4. (a) Cross sectional schematic of a CMOS inverter fabricated by connecting $\mathrm{n}$ - and p-type nanotube TFTs on the same substrate as obtained by patterned deposition of $\mathrm{SiN}_{x}$ thin films. (b) Transfer characteristics of a CMOS nanotube inverter showing a gain of $\sim 6.2$.
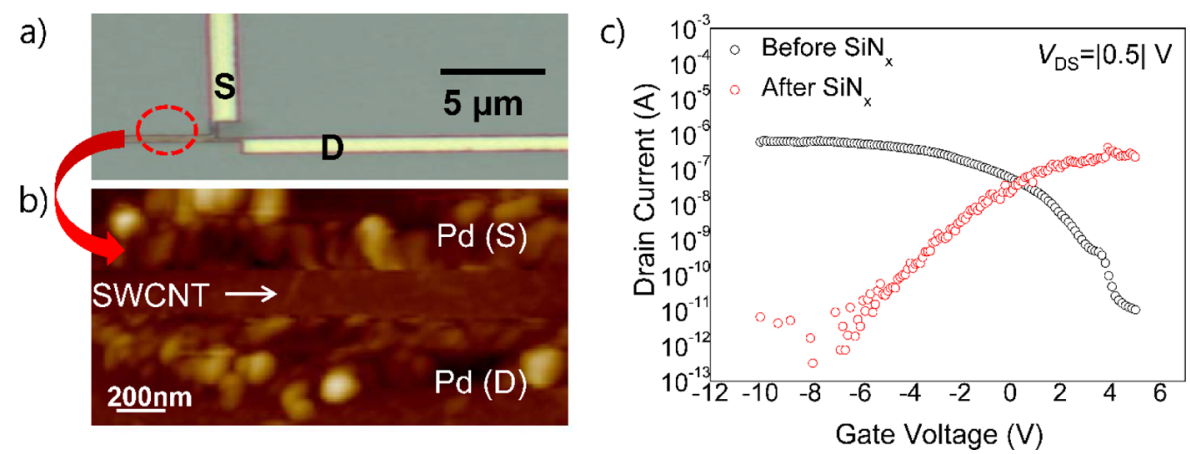

Figure 5. Single nanotube devices. (a) Optical microscope and (b) AFM images of a back-gated transistor based on an individual SWCNT. (c) Transfer characteristics of the device before and after $\mathrm{SiN}_{x}$ deposition.

after doping with $\mathrm{SiN}_{x}$. Pd has been shown previously to exhibit one of the lowest contact resistances to metallic nanotubes due to its strong wettability with SWCNTs. ${ }^{2,41,42}$ Given that electron injection in $\mathrm{SiN}_{x}$ coated nanotube TFTs is obtained by tunnelling through the thinned SBs at the contacts rather than thermionic emission over the barrier, the contact resistance is largely dependent on the width of the SBs and the interface quality (i.e., metal wettability ${ }^{41,42}$ ). While the former depends on the doping concentration, the latter depends on the choice of the metal contact. In this regard, similar to the case of metallic nanotubes, Pd offers the best interface with SWCNTs. ${ }^{26,43}$

$\mathrm{SiN}_{x}$ stoichiometry affects the obtained fixed charge density. ${ }^{35}$ Film stoichiometry and hydrogen content were determined by Rutherford backscattering spectrometry (RBS) and hydrogen forward scattering (HFS) using a $2.79 \mathrm{MeV}$ alpha beam. RBS measurements were carried out at a backscattered angle of $165^{\circ}$. Quantitative hydrogen profiling was performed by HFS in a forward scattering geometry with the detector positioned at $150^{\circ}$ with respect to the incident beam. The sample was tilted so that the sample normal made a $75^{\circ}$ angle with the incident beam and a $13 \mu \mathrm{m}$ thick Mylar foil was placed in front of the detector to stop the scattered alphas. The collected spectra were analyzed using the SIMNRA software code. ${ }^{44}$ From RBS (Figure $3 \mathrm{a}$ ) and HFS measurements (Figure $3 \mathrm{~b}$ ), we observed that $\mathrm{SiN}_{x}$ films are hydrogenated with a hydrogen areal density of $2.75 \times 10^{18}$ atoms/ $\mathrm{cm}^{2}$ corresponding to a film stoichiometry of $\mathrm{Si}_{0.39} \mathrm{~N}_{0.35} \mathrm{H}_{0.26}$. This composition is in line with literature for the deposition parameters used. $^{45}$

To determine the fixed charge density within this nitride film, capacitance-voltage $(C-V)$ measurements were carried out on a metal-insulator-semiconductor structure of $\mathrm{Al}, \mathrm{PECVD}$ $\mathrm{SiN}_{x}$, and n-type $\mathrm{Si}$ (resistivity of $10-30 \Omega \mathrm{cm}$ ) as shown in Figure 3c. The device area is $\sim 650 \times 650 \mu \mathrm{m}^{2}$. The fixed charge density, $Q_{F}$, can be extracted from the flatband voltage of the device using the equation $Q_{\mathrm{F}}=C_{\mathrm{SiN}_{x}}\left(\phi_{\mathrm{MS}}-V_{\mathrm{FB}}\right) / q$ where $C_{\mathrm{SiN}_{x}}$ is the accumulation capacitance, $\phi_{\mathrm{MS}} \sim-0.23 \mathrm{~V}$ is the work function difference between $\mathrm{Al}$ and the $\mathrm{Si}$ wafer, $q$ is the elemental unit of charge, and $V_{\mathrm{FB}}$ is the flatband voltage. A $V_{\mathrm{FB}}$ of $-4.4 \mathrm{~V}$ was extracted from the second derivative of the measured $C-V$ plot, corresponding to the peak of $d^{2}\left(1 / C_{\mathrm{SiN} x}^{2}\right) /$ $d V^{2}$. Using this analysis, the fixed charge density in the nitride is $\sim 7 \times 10^{11} \mathrm{~cm}^{-2}$, which is in line with the reported values in literature. ${ }^{45}$ This fixed charge must be compensated by the carbon nanotube film with an effective coverage area of $4-9 \%$ as approximated via AFM images. Assuming that the film is approximately a monolayer of SWCNTs with average diameter of $1.5 \mathrm{~nm}$, the doping level induced by the silicon nitride fixed charge is on the order of $5 \times 10^{19}$ to $1 \times 10^{20} \mathrm{~cm}^{-3}$.

Given the near symmetric electrical properties of $n$ - and $p$ type SWCNT TFTs and high device-to-device uniformity for both polarities, CMOS circuitry can be explored. As a proof-ofconcept, a CMOS inverter using a p-type TFT (i.e., without $\mathrm{SiN}_{x}$ ) and an n-type TFT obtained by $\mathrm{SiN}_{x}$ coating is fabricated as schematically illustrated in Figure 4a. Patterned $\mathrm{SiN}_{x}$ deposition was obtained during PECVD by using a shadow mask, thus providing a simple and facile approach for fabricating p-and n-type SWCNT-TFTs on the same substrate. Figure $4 \mathrm{~b}$ shows the transfer characteristics of the CMOS inverter, exhibiting a maximum DC gain of $\sim 6.2$ at an operation voltage of $4 \mathrm{~V}$ with a noise margin of $1.68 \mathrm{~V}$. In the future, more sophisticated CMOS circuits can be obtained by fabricating transistors with local back-gates. 
$\mathrm{SiN}_{x}$ field-effect doping is also tested on single nanotube devices in addition to the random network TFTs discussed above. Single SWCNT FETs were fabricated by spin-coating the semiconductor-enriched nanotube suspension onto the poly-L-lysine modified $\mathrm{SiO}_{2}$, resulting in a low density of assembled nantoubes on the surface. AFM was used to map the surface and locate inividual SWCNTs in respect to prefabricated alignment marks. Pd S/D contacts were subsequently fabricated on individual SWCNTs using electron-beam lithography. Figure 5a,b shows the optical and AFM images of a device consisting of a single tube (diameter, $\sim 1.4$ $\mathrm{nm}$ ), respectively. The channel length (i.e., S/D spacing) for this device is $\sim 200 \mathrm{~nm}$. As expected, the device without $\mathrm{SiN}_{x}$ coating exhibits p-type switching characteristics (Figure 5c). After $\mathrm{SiN}_{x}$ deposition, polarity conversion to n-type is observed with similar ON currents for both cases. Furthermore, $I_{\mathrm{ON}} / I_{\mathrm{OFF}}$ $>10^{5}$ is observed for both cases. The results suggest that $\mathrm{SiN}_{x}$ field-effective doping is also compatible with single nanotube devices. Importantly, the similar ON-state current for both polarities suggests that the nanotube quality remains pristine after $\mathrm{SiN}_{x}$ deposition, with PECVD processing not inducing defects in SWCNTs.

In conclusion, a new air-stable doping concept for SWCNTs is demonstrated by using $\mathrm{SiN}_{x}$ thin films deposited by PECVD. The fixed positive charges within $\mathrm{SiN}_{x}$ act to $\mathrm{n}$-dope the underlying nanotubes via field-effect, which is in distinct contrast to the charge transfer doping commonly explored in the past. Given the large knowledge base and infrastructure built around $\mathrm{SiN}_{x}$ thin film passivation in the silicon solar cell industry, this doping concept presents a reliable and easy to process route toward obtaining high performance SWCNT ntype transistors. Specifically, the obtained n-type SWCNT TFTs exhibit excellent device-to-device uniformity with comparable electrical characteristics to their p-type counterparts. As a proof of concept, a CMOS invertor is demonstrated using two SWCNT TFTs. In the future, the doping level of SWCNTs can be tuned by changing the stoichiometry of the nitride by changing PECVD process parameters, thus changing the fixed charge density. Furthermore, this doping concept can be extended to p-doping of carbon nanotubes by using other insulators with fixed negative charges. The work presents an important advance toward realization of CMOS circuitry based on nanotube TFTs for large-area electronic applications, where random networks of SWCNTs have shown to be a promising material system.

\section{ASSOCIATED CONTENT}

\section{S Supporting Information}

$\mathrm{SiN}_{x}$ thickness dependence on device operation; bias stress testing of TFTs with and without $\mathrm{SiN}_{x}$ thin film; and contact metal dependence on TFTs after $\mathrm{SiN}_{x}$ deposition. This material is available free of charge via the Internet at http://pubs.acs.org.

\section{AUTHOR INFORMATION}

\section{Corresponding Author}

*E-mail: ajavey@eecs.berkeley.edu.

\section{Present Address}

(T.-J.H.) Department of Electronic Materials Engineering, Kwangwoon University, Seoul 139-701, Republic of Korea.

\section{Author Contributions}

T.-J.H. and K.C. contributed equally to this work.

\section{Notes}

The authors declare no competing financial interest.

\section{ACKNOWLEDGMENTS}

This work was supported by NSF NASCENT Center. $\mathrm{SiN}_{x}$ characterization, including RBS, was supported by the Director, Office of Science, Office of Basic Energy Sciences, Material Sciences and Engineering Division, U.S. Department of Energy, under Contract DE-AC02-05CH11231.

\section{REFERENCES}

(1) Sangwan, V. K.; Ortiz, R. P.; Alaboson, J. M. P.; Emery, J. D.; Bedzyk, M. J.; Lauhon, L. J.; Marks, T. J.; Hersam, M. C. ACS Nano 2012, 6, 7480-7488.

(2) Javey, A.; Guo, J.; Wang, Q.; Lundstrom, M.; Dai, H. Nature 2003, 424, 654-657.

(3) Dürkop, T.; Getty, S. A.; Cobas, E.; Fuhrer, M. S. Nano Lett. 2004, 4, 35-39.

(4) Shulaker, M. M.; Hills, G.; Patil, N.; Wei, H.; Chen, H.-Y.; Wong, H.-S. P.; Mitra, S. Nature 2013, 501, 526-530.

(5) Wang, C.; Zhang, J.; Ryu, K.; Badmaev, A.; De Arco, L. G.; Zhou, C. Nano Lett. 2009, 9, 4285-4291.

(6) Kiriya, D.; Chen, K.; Ota, H.; Lin, Y.; Zhao, P.; Yu, Z.; Ha, T.; Javey, A. J. Am. Chem. Soc. 2014, 136, 11188-11194.

(7) Wang, C.; Takei, K.; Takahashi, T.; Javey, A. Chem. Soc. Rev. 2013, 42, 2592.

(8) Lau, P. H.; Takei, K.; Wang, C.; Ju, Y.; Kim, J.; Yu, Z.; Takahashi, T.; Cho, G.; Javey, A. Nano Lett. 2013, 13, 3864-3869.

(9) Chen, P.; Fu, Y.; Aminirad, R.; Wang, C.; Zhang, J.; Wang, K.; Galatsis, K.; Zhou, C. Nano Lett. 2011, 11, 5301-5308.

(10) Ha, M.; Xia, Y.; Green, A. A.; Zhang, W.; Renn, M. J.; Kim, C. H.; Hersam, M. C.; Frisbie, C. D. ACS Nano 2010, 4, 4388-4395.

(11) Jung, M.; Kim, J.; Noh, J.; Lim, N.; Lim, C.; Lee, G.; Kim, J.; Kang, H.; Jung, K.; Leonard, A. D.; Tour, J. M.; Cho, G. IEEE Trans. Electron Devices 2010, 57, 571-580.

(12) Noh, J.; Jung, M.; Jung, K.; Lee, G.; Kim, J.; Lim, S.; Kim, D.; Choi, Y.; Kim, Y.; Subramanian, V.; Cho, G. IEEE Electron Device Lett. 2011, 32, 638-640.

(13) Wang, C.; Hwang, D.; Yu, Z.; Takei, K.; Park, J.; Chen, T.; Ma, B.; Javey, A. Nat. Mater. 2013, 12, 899-904.

(14) Zhang, J.; Wang, C.; Zhou, C. ACS Nano 2012, 6, 7412-7419.

(15) Che, Y.; Badmaev, A.; Jooyaie, A.; Wu, T.; Zhang, J.; Wang, C.; Galatsis, K.; Enaya, H. A.; Zhou, C. ACS Nano 2012, 6, 6936-6943.

(16) Ding, J.; Li, Z.; Lefebvre, J.; Cheng, F.; Dubey, G.; Zou, S.; Finnie, P.; Hrdina, A.; Scoles, L.; Lopinski, G. P.; Kingston, C. T.; Simard, B.; Malenfant, P. R. L. Nanoscale 2014, 6, 2328-2339.

(17) Zhang, J.; Fu, Y.; Wang, C.; Chen, P.-C.; Liu, Z.; Wei, W.; Wu, C.; Thompson, M. E.; Zhou, C. Nano Lett. 2011, 11, 4852-4858.

(18) Takahashi, T.; Yu, Z.; Chen, K.; Kiriya, D.; Wang, C.; Takei, K.; Shiraki, H.; Chen, T.; Ma, B.; Javey, A. Nano Lett. 2013, 13, 54255430.

(19) Yasunishi, T.; Kishimoto, S.; Ohno, Y. Jpn. J. Appl. Phys. 2014, 53, 05 FD01.

(20) Shahrjerdi, D.; Franklin, A. D.; Oida, S.; Ott, J. A.; Tulevski, G. S.; Haensch, W. ACS Nano 2013, 7, 8303-8308.

(21) Ding, L.; Wang, S.; Zhang, Z.; Zeng, Q.; Wang, Z.; Pei, T.; Yang, L.; Liang, X.; Shen, J.; Chen, Q.; Cui, R.; Li, Y.; Peng, L.-M. Nano Lett. 2009, 9, 4209-4214.

(22) Yang, L.; Wang, S.; Zeng, Q.; Zhang, Z.; Pei, T.; Li, Y.; Peng, L.M. Nat. Photonics 2011, 5, 672-676.

(23) Javey, A.; Wang, Q.; Ural, A.; Li, Y.; Dai, H. Nano Lett. 2002, 2, 929-932.

(24) Shim, M.; Javey, A.; Kam, N. W.; Dai, H. J. Am. Chem. Soc. 2001, $123,11512-11513$.

(25) Kim, S. M.; Jang, J. H.; Kim, K. K.; Park, H. K.; Bae, J. J.; Yu, W. J.; Lee, I. H.; Kim, G.; Loc, D. D.; Kim, U. J.; Lee, E.-H.; Shin, H.-J.; Choi, J.-Y.; Lee, Y. H. J. Am. Chem. Soc. 2009, 131, 327-331. 
(26) Javey, A.; Tu, R.; Farmer, D. B.; Guo, J.; Gordon, R. G.; Dai, H. Nano Lett. 2005, 5, 345-348.

(27) Wang, C.; Ryu, K.; Badmaev, A.; Patil, N.; Lin, A.; Mitra, S.; Wong, H.-S. P.; Zhou, C. Appl. Phys. Lett. 2008, 93, 033101.

(28) Kishimoto, T.; Ohno, Y.; Maehashi, K.; Inoue, K.; Matsumoto, K. Jpn. J. Appl. Phys. 2010, 49, 06GG02.

(29) Liang, S.; Zhang, Z.; Pei, T.; Li, R.; Li, Y.; Peng, L. Nano Res. 2013, 6, 535-545.

(30) Suriyasena Liyanage, L.; Xu, X.; Pitner, G.; Bao, Z.; Wong, H.-S. P. Nano Lett. 2014, 14, 1884-1890.

(31) Zhang, J.; Wang, C.; Fu, Y.; Che, Y.; Zhou, C. ACS Nano 2011, 5, 3284-3292.

(32) Franklin, A. D.; Koswatta, S. O.; Farmer, D. B.; Smith, J. T.; Gignac, L.; Breslin, C. M.; Han, S.-J.; Tulevski, G. S.; Miyazoe, H.; Haensch, W.; Tersoff, J. Nano Lett. 2013, 13, 2490-2495.

(33) Robertson, J. Philos. Mag., B 1994, 69, 307-326.

(34) Hezel, R.; Blumenstock, K.; Schörner, R. J. Electrochem. Soc. 1984, 131, 1679-1683.

(35) Chen, K.; Kiriya, D.; Hettick, M.; Tosun, M.; Ha, T.-J.; Madhvapathy, S. R.; Desai, S.; Sachid, A.; Javey, A. APL Mater. 2014, 2, 092504.

(36) Mäckel, H.; Lüdemann, R. J. Appl. Phys. 2002, 92, 2602-2609.

(37) Aberle, A. G. Crystalline Silicon Solar Cells: Advanced Surface Passivation and Analysis; Centre for Photovoltaic Engineering, University of New South Wales: Sydney, Austrailia, 1999.

(38) Schorner, R.; Hezel, R.; Hezel, R. IEEE Trans. Electron Devices 1981, 28, 1466-1469.

(39) Lauinger, T.; Schmidt, J.; Aberle, A. G.; Hezel, R. Appl. Phys. Lett. 1996, 68, 1232-1234.

(40) Aberle, A. G. Sol. Energy Mater. Sol. Cells 2001, 65, 239-248.

(41) Zhang, Y.; Dai, H. Appl. Phys. Lett. 2000, 77, 3015-3017.

(42) Shan, B.; Cho, K. Phys. Rev. B 2004, 70, 233405.

(43) Mann, D.; Javey, A.; Kong, J.; Wang, Q.; Dai, H. Nano Lett. 2003, 3, 1541-1544.

(44) Mayer, M. AIP Conf. Proc. 1999, 475, 541-544.

(45) Haug, H.; Klingshirn, C.; Landolt, H.; Börnstein, R. 2001; pp $1524-1529$. 


\section{Highly uniform and stable $n$-type carbon nanotube transistors by using positively charged silicon nitride thin films}

Tae-Jun $\mathrm{Ha}^{1,2,+, \dagger}$, Kevin Chen ${ }^{1,2,+}$, Steven Chuang ${ }^{1,2}$, Kin Man $\mathrm{Yu}^{2}$, Daisuke Kiriya ${ }^{1,2}$, Ali Javey ${ }^{1,2,{ }^{*}}$

${ }^{1}$ Electrical Engineering and Computer Sciences, University of California, Berkeley, CA 94720

${ }^{2}$ Materials Sciences Division, Lawrence Berkeley National Laboratory, Berkeley, CA 94720

*Corresponding Author: ajavey@eecs.berkeley.edu

${ }^{+}$These authors contributed equally to this work

$\dagger$ Present address: Department of Electronic Materials Engineering, Kwangwoon University, Seoul 139-701, Republic of Korea

\section{Supporting Information}




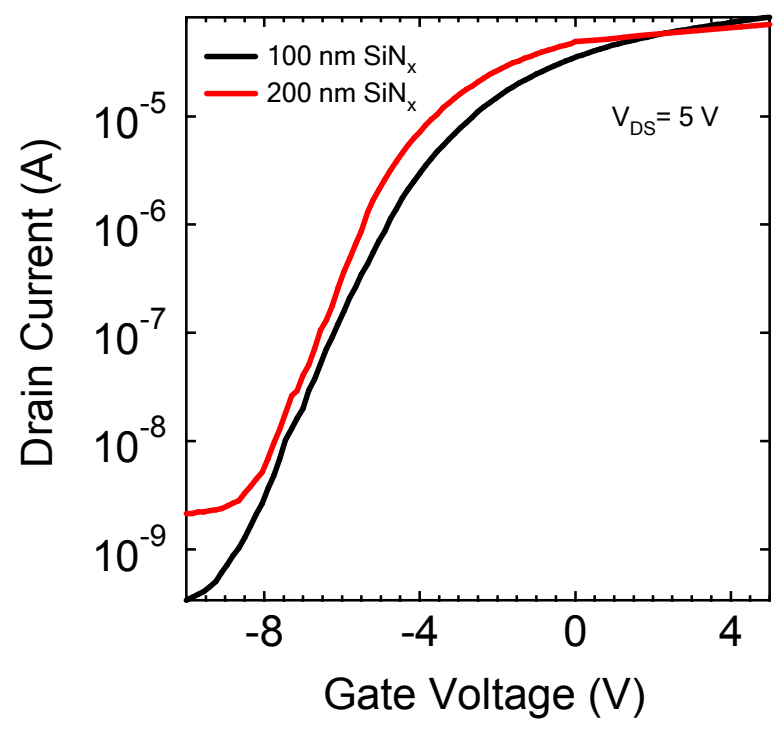

Figure S1: Transfer characteristics of a representative SWCNT TFT after $100 \mathrm{~nm}$ of $\mathrm{SiN}_{\mathrm{x}}$ thin film deposition (black curve) and after an additional $100 \mathrm{~nm}$ of $\operatorname{SiN}_{\mathrm{x}}$ (total 200 $\mathrm{nm}$; red curve), showing minimal difference in the transfer characteristics. 


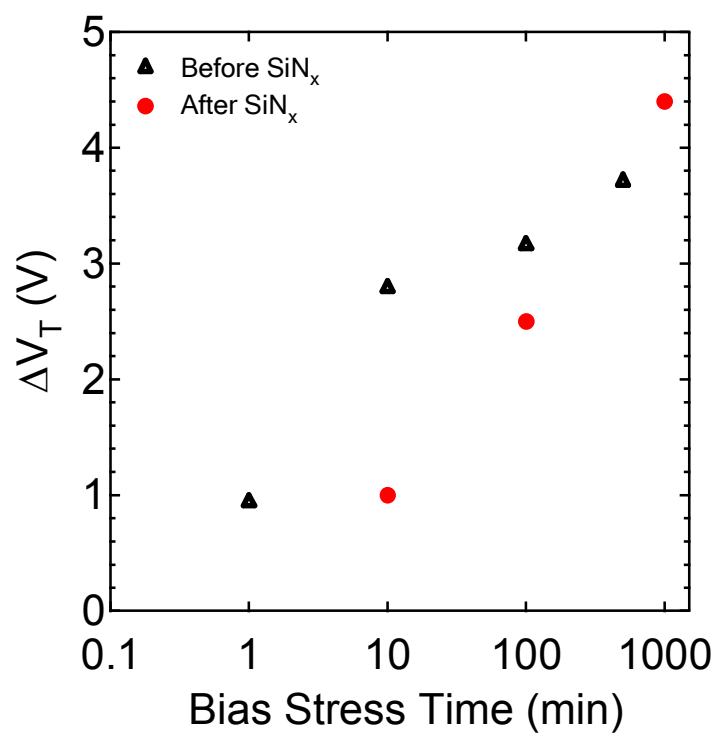

Figure S2: Change in the threshold voltage versus the initial zero stress threshold voltage after bias stress testing of representative SWCNT TFTs before $\mathrm{SiN}_{\mathrm{x}}$ deposition and after. The devices were placed under a bias stress of $10 \mathrm{~V}$ gate voltage and $10 \mathrm{~V}$ drain voltage. 


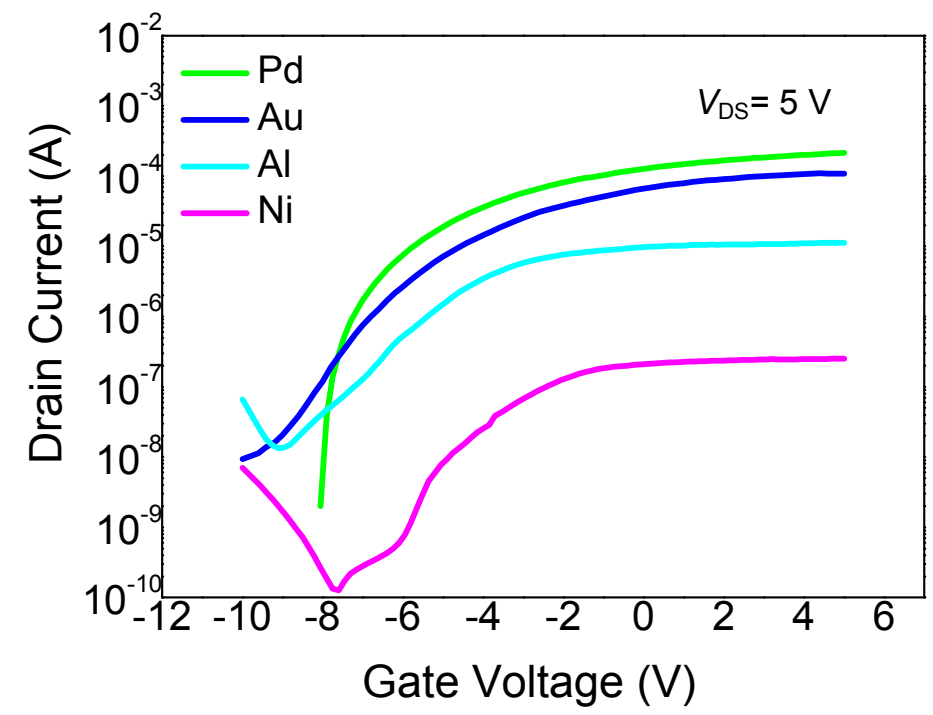

Figure S3: Transfer characteristics of SWCNT TFTs after $\mathrm{SiN}_{\mathrm{x}}$ deposition with $\mathrm{Pd}, \mathrm{Au}$, Al, and Ni S/D contacts. Regardless of the metal contact, the SWCNT devices all exhibit n-type behavior after $\mathrm{SiN}_{\mathrm{x}}$ with Pd exhibiting highest $\mathrm{ON}$-state current, corresponding to best SWCNT/metal interface. 